ORIGINAL RESEARCH

\title{
Baseline Competency Assessment of Pharmacists Prescribing and Managing Vancomycin Therapy in the Regina Qu'Appelle Health Region
}

\author{
Kirsten Tangedal, Jennifer Bolt, Suzanne Len, and Ali Bell
}

\begin{abstract}
Background: Pharmacists in the Regina Qu'Appelle Health Region (RQHR), Saskatchewan, independently dose, monitor, and adjust vancomycin therapy. No framework exists for ongoing competency assessment of pharmacists.

Objectives: The primary objective was to determine pharmacists' overall level of competency for all components of the vancomycin prescribing procedure. The secondary objectives were to determine competency for individual prescribing phases, to stratify overall competency in relation to pharmacist and patient factors, and to identify the 3 most frequent errors.

Methods: A retrospective chart audit was performed of patients who received a prescription for vancomycin between November 1, 2015, and January 31, 2016. Patients were included if they received pharmacistprescribed vancomycin as an inpatient or outpatient of an RQHR facility. Patients under the care of a pediatrician, those receiving vancomycin for surgical prophylaxis or via any route other than the IV route, and those whose vancomycin was prescribed by a current pharmacy resident were excluded. A rubric was created that assigned a numeric value for the appropriate completion of various procedure criteria.

Results: A total of 326 patients received vancomycin during the study period, of whom 200 met the inclusion criteria, representing 511 discrete episodes of prescribing by 42 pharmacists. The median overall competency rate, for all phases of prescribing, was 100\% (interquartile range [IQR] 90.1\%-100\%). The median competency rates for the empiric therapy and monitoring phases were $94.4 \%$ (IQR $88.9 \%-100 \%$ ) and $100 \%$ (IQR $87.5 \%-100 \%$ ), respectively. No statistically significant differences were found in relation to pharmacists' experience or postbaccalaureate education, patients' level of acuity, or timing of prescribing. The competency score was significantly higher among pharmacists prescribing for patients with normal renal function than among those prescribing for patients with reduced renal function $(p=0.008)$. The 3 most common errors were failure to document risk factors for nephrotoxicity, failure to document requirement to obtain future trough levels, and failure to document that samples for trough levels had been drawn correctly.
\end{abstract}

Conclusions: During the study period, pharmacists at RQHR showed competency in all phases of vancomycin prescribing using the approved

\section{RÉSUMÉ}

Contexte : Des pharmaciens de la régie régionale de la santé de Regina Qu'Appelle (RRSRQ) en Saskatchewan s'occupent eux-mêmes de doser la vancomycine ainsi que d'en surveiller et d'en ajuster la posologie. Or, à ce jour, aucun cadre n'entoure l'évaluation continue de la compétence de ces pharmaciens.

Objectifs : L'objectif principal était de déterminer le niveau global de compétence des pharmaciens pour tous les éléments de la marche à suivre pour prescrire la vancomycine. Les objectifs secondaires consistaient à déterminer le niveau de compétence pour chaque étape de la prescription, à stratifier le niveau global de compétence en fonction de facteurs se rapportant au pharmacien et au patient et à identifier les trois erreurs les plus courantes.

Méthodes : On a réalisé une vérification rétrospective des dossiers médicaux de patients qui se sont fait prescrire la vancomycine entre le $1^{\text {er }}$ novembre 2015 et le 31 janvier 2016. Les patients admis à l'étude devaient avoir reçu la vancomycine sur la prescription d'un pharmacien alors qu'ils étaient hospitalisés ou en consultation externe dans un établissement de la RRSRQ. Les patients soignés par un pédiatre, ceux ayant reçu un traitement prophylactique de vancomycine pour une intervention chirurgicale, ceux ayant reçu le médicament autrement que par voie intraveineuse et ceux dont la vancomycine a été prescrite par un résident en pharmacie à l'époque ont été exclus. Une grille d'évaluation a été créée afin d'accorder une valeur numérique selon le degré de conformité de l'exécution aux différents critères de la marche à suivre.

Résultats : Au total, 326 patients ont reçu la vancomycine pendant la période d'étude. Parmi eux, 200 répondaient aux critères d'inclusion, ce qui représentait 511 actes distincts de prescription réalisés par 42 pharmaciens. Le taux de compétence global médian pour toutes les phases de la prescription était de $100 \%$ (écart interquartile [ÉIQ] de 90,1\% à $100 \%$ ). Les taux de compétence médians pour les phases de l'antibiothérapie empirique et du suivi étaient respectivement de 94,4\% (ÉIQ de $88,9 \%$ à $100 \%$ ) et de $100 \%$ (ÉIQ de $87,5 \%$ à $100 \%$ ). Aucune différence statistiquement significative quant à la compétence n’a été relevée par rapport à l'expérience du pharmacien, aux études universitaires de cycles supérieurs, à la gravité de l'état du patient ou au moment de la réalisation de la prescription. Le score de compétence était significativement plus élevé chez les pharmaciens prescrivant à des patients dont 
procedure. Documentation of clinical plans and assessments was identified as an area for improvement.

Keywords: vancomycin, pharmacist, competency, assessment

\section{Can J Hosp Pharm. 2017;70(5):335-42}

la fonction rénale est normale que pour ceux prescrivant à des patients atteints d'insuffisance rénale $(p=0,008)$. Les trois erreurs les plus courantes étaient : négliger de consigner les facteurs de risque néphrotoxique, négliger de consigner que l'obtention de futures concentrations minimales était nécessaire et négliger de consigner que les échantillons pour les concentrations minimales avaient été prélevés correctement.

Conclusions : Pendant la période d'étude, les pharmaciens travaillant à la RRSRQ ont fait preuve de compétence dans l'ensemble des phases de prescription de la vancomycine en utilisant la marche à suivre approuvée. On a noté qu'il fallait améliorer la consignation des plans cliniques et des évaluations.

Mots clés : vancomycine, pharmacien, compétence, évaluation

\section{INTRODUCTION}

$\mathrm{V}$ ancomycin is a glycopeptide antibiotic that exhibits time-dependent bactericidal activity. ${ }^{1}$ It is a first-line treatment for infections caused by methicillin-resistant Staphylococcus aureus (MRSA), according to the 2011 Infectious Diseases Society of America guidelines for the treatment of MRSA infections. ${ }^{2}$

Vancomycin has a narrow therapeutic range. ${ }^{3}$ Subtherapeutic concentrations (i.e., below $10 \mathrm{mg} / \mathrm{L}$ ) are associated with potential treatment failure, whereas supratherapeutic levels (i.e., above $20 \mathrm{mg} / \mathrm{L}$ ) are associated with potential toxicity, including nephrotoxicity. ${ }^{2}$ As a result, vancomycin dosing must be individualized. Empiric dosing is based on weight, renal function, and indication for therapy. ${ }^{2}$ After therapy has been initiated, steady-state trough levels are used to guide adjustments to the dosing regimen, to ensure that concentrations are within the desired therapeutic range. ${ }^{2}$ Sampling at appropriate times for the determination of trough levels is critical to vancomycin dosing; delayed or early sampling may result in inappropriate dosing decisions. ${ }^{4}$

An often-feared adverse effect associated with vancomycin therapy is nephrotoxicity. Vancomycin-induced nephrotoxicity is defined as a $50 \%$ or greater relative increase in serum creatinine or an absolute increase in serum creatinine of $40 \mu \mathrm{mol} / \mathrm{L}$ or greater above baseline. The effect must occur on 2 consecutive days after several days of vancomycin therapy and must be in the absence of another explanation. ${ }^{5-7}$ Rates of vancomycin nephrotoxicity are variable across studies (from $5 \%$ to $43 \%$ ), and of those who experience nephrotoxicity only $3 \%$ require short-term dialysis. ${ }^{6}$ The requirement for long-term dialysis due to vancomycin nephrotoxicity has not been reported. ${ }^{6}$

Clinical pharmacists can play a significant role in the safe and effective use of vancomycin therapy. ${ }^{8}$ Marquis and others ${ }^{9}$ evaluated the effectiveness of a pharmacist-directed vancomycin dosing and monitoring pilot program by assessing the proportion of patients who received the correct weight-based dose. This prepost intervention study analyzed the vancomycin therapy of 319 patients (161 pre-implementation and 158 post-implementation) and found that the percentage of patients who received optimal vancomycin dosing was significantly higher after implementation of pharmacist-directed prescribing (96.8\% versus $40.4 \%$, $p<0.001) .{ }^{9}$ The authors also found a shorter length of therapy (8.4 versus 10.0 days, $p<0.003$ ) and a lower incidence of nephrotoxicity $(3.2 \%$ versus $8.7 \%, p<0.001)$ among patients for whom vancomycin was prescribed by a pharmacist relative to prescribing by a physician. ${ }^{9}$ Masuda and others ${ }^{10}$ assessed the effectiveness of pharmacist-directed vancomycin therapy in a pre-post intervention study by looking at time spent within the therapeutic range. Their study included 610 patients $(508$ pre-intervention, 102 post-intervention) and showed that pharmacist-directed vancomycin therapy resulted in a significant increase in time spent in the desired therapeutic range $(10-20 \mathrm{mg} / \mathrm{L})$ relative to physician-directed therapy $(p<0.001) .{ }^{10}$ This study also found a trend toward a decrease in nephrotoxicity in the pharmacistdirected therapy cohort. ${ }^{10}$

In the Regina Qu'Appelle Health Region (RQHR), Saskatchewan, about 100 patients per month receive vancomycin. Pharmacists and physicians within RQHR have a collaborative practice agreement that gives pharmacists the authority to dose, monitor, and adjust vancomycin therapy that has been initiated by a physician. Pharmacists undergo initial training and competency assessment through a certification examination to ensure compliance with the prescribing procedure. Although some quality assessment of supratherapeutic trough levels is performed, RQHR currently lacks a standardized method to evaluate ongoing competency with respect to vancomycin prescribing. A new vancomycin protocol and procedure were implemented in September 2015 in the RQHR, and development of competency standards is necessary to ensure that pharmacists are competently prescribing the drug according to the new procedure. Key changes in the procedure include implementation of loading doses, changes to dosing intervals, documentation of patient-specific risk factors for nephrotoxicity, and documentation of duration of therapy. The purpose of this study was to assess the competency of pharmacists within RQHR in terms of the skills of vancomycin dosing, monitoring, and therapy adjustment. 
The primary objective was to determine the mean level of overall pharmacist competency, on the basis of all components of the vancomycin prescribing procedure. The secondary objectives were to identify the most common errors in prescribing and to determine the mean level of competency for the following:

- empiric dosing of vancomycin

- vancomycin monitoring and dose adjustment

- new staff (<2 years' experience at RQHR), staff with mid-range experience (2-5 years), and staff with the most experience $(>5$ years)

- pharmacists with and without postbaccalaureate training (Accredited Canadian Pharmacy Residency, Doctor of Pharmacy, or Clinical Master's)

- pharmacists treating patients with normal renal function (creatinine clearance $[\mathrm{CrCl}]$ greater than or equal to $60 \mathrm{~mL} / \mathrm{min})$ and reduced renal function $(\mathrm{CrCl}$ less than $60 \mathrm{~mL} / \mathrm{min}$ )

- pharmacists treating patients in critical care units, patients in non-critical care units, and outpatients

- various staffing levels at time of prescribing

\section{METHODS}

A retrospective chart audit was conducted to assess pharmacist competency in using the RQHR vancomycin procedure introduced in September 2015. Patients eligible for inclusion were those for whom vancomycin was prescribed by a pharmacist within an RQHR facility in Regina, Saskatchewan (Regina General Hospital, Pasqua Hospital, or Wascana Rehabilitation Centre), from November 1, 2015, to January 31, 2016. The patient population for these facilities included inpatients, emergency department outpatients, hemodialysis outpatients, and patients of the home IV therapy program. Patients were excluded if they had received vancomycin by a route other than intermittent IV infusion (e.g., peritoneal, oral); if they had received vancomycin for surgical prophylaxis; if they were under the care of a pediatrician, neonatologist, or pediatric oncologist; or if vancomycin had been prescribed outside of the approved protocol (termed "off-protocol prescribing"). In contrast to prescriptive authority, whereby pharmacists can independently write orders for vancomycin dosing and monitoring, off-protocol prescribing requires that pharmacists discuss their recommendations with the prescribing physician, who then provides a verbal or written medication order. For patients who received multiple discrete courses of vancomycin during a single hospital visit, only the index course (i.e., the first course of therapy) was included in the study. Patients with a diagnosis of febrile neutropenia were also excluded, because there was a revision in procedure for this population midway through the data collection period. If vancomycin had been initiated at a different health care centre, the patient's data were not included until management according to the RQHRapproved vancomycin procedure began. These exclusion criteria are summarized in Box 1.

\section{Box 1. Exclusion Criteria}

Vancomycin administered through any route other than intermittent IV infusion (e.g., peritoneal, oral)

Surgical prophylaxis

Patient under the care of a pediatrician, neonatologist, or pediatric oncologist

Vancomycin prescribed outside of the approved protocol

Patient with febrile neutropenia

Prescribing done by a current pharmacy resident

Subsequent initiation(s) of vancomycin in the same patient

Pharmacists eligible for inclusion were those who had successfully completed the initial certification process for vancomycin prescribing, as implemented at the time of the procedure update in September 2015. The initial certification process consisted of two 1-h sessions of training or a review of prerecorded training sessions, followed by a certification exam. Pharmacists who were pharmacy residents at the time of the study were excluded, because of the possible effect of a preceptor on prescribing by a resident. Eligible patients were identified through BDM Pharmacy (BDM IT Solutions, Saskatoon, Saskatchewan), the medication management software used by the RQHR Pharmacy Department. A report was generated listing all patients who received at least one dose of vancomycin during the study period. For all patients who met the inclusion criteria, vancomycin prescribing was evaluated from initiation to discontinuation with use of a rubric created specifically for this study (as described below). The following data were collected for each patient: location at the time of prescribing (critical care unit [medical intensive care unit, surgical intensive care unit, coronary care unit], non-critical care unit [emergency department, all other inpatient units], outpatient setting, or home [for long-term IV therapy]), serum creatinine level at initiation of vancomycin therapy, and day of the week and time of day of prescribing (Monday-Friday, 0800-1630; Monday-Friday, 1630-2030; or weekend or statutory holiday) for each episode of prescribing. For prescribing done via phone order by an on-call pharmacist, the time of day was recorded as the time when the empiric consult was completed. Patient-specific data related to vancomycin prescribing were also collected, including indication for therapy, target trough level, loading dose, maintenance dose, dosing interval, risk factors for nephrotoxicity as defined by the prescribing procedure (serum creatinine $\geq 100 \mu \mathrm{mol} / \mathrm{L}$, weight $\geq 100 \mathrm{~kg}$ or morbid obesity, hypotension due to septic shock [receiving vasopressors], target trough $15-20 \mathrm{mg} / \mathrm{L}$, daily dose $\geq 4 \mathrm{~g}$ ), duration of vancomycin therapy, frequency of serum creatinine monitoring, serum creatinine level (used to estimate creatinine clearance with the modified Cockcroft-Gault equation ${ }^{11,12}$ ), and serum vancomycin levels. These data were collected from pharmacists' documentation notes in the progress notes section and the physician's orders section of patients' medical records.

A validated auditing tool was not available to evaluate pharmacists' competency in prescribing vancomycin. Therefore, 
the primary investigator (K.T.) drafted a rubric based on all components of the RQHR "Pharmacist's Procedure Re: Vancomycin Protocol - Treatment in Adults" (revised August 13, 2015). Each step of the procedure was assessed and a checklist made of all the criteria that a pharmacist must complete. Assessment and preparation of a checklist were completed for both the empiric and maintenance phases of prescribing and for all populations covered by the procedure (adults, adults with acute renal failure, and adults with chronic renal failure). The rubric had specific sections for the various aspects of prescribing (empiric phase, trough level, serum creatinine, and duration of follow-up). Points were applied for each criterion, with weighting of scores according to the potential impact of an error on the patient (e.g., more points lost for a dosing error than for monitoring serum creatinine too frequently). Certain sections of the rubric, such as those for maintenance dose and dosing interval, were weighted in such a way that errors in these categories would prevent the pharmacist from reaching the predefined threshold for competency. Errors that would result in the pharmacist being scored as "incompetent", deemed "critical errors", were decided upon by consensus among the investigators. The point system and rubric content were assessed by each co-investigator, as well as a staff pharmacist external to the study, to ensure that the weighting system accurately calculated competency scores (Appendix 1, available at www.cjhp-online.ca/index.php/cjhp/ issue/view/123/showToc).

During development of the rubric, the primary investigator (K.T.) applied the rubric to 5 episodes of empiric prescribing and 5 episodes of prescribing for the monitoring phase to evaluate applicability. Feedback from one of the co-investigators (S.L.) was used to make minor changes before the official data collection period. The rubric was also pilot-tested before the data collection period, with the primary investigator and the same co-investigator independently applying the rubric to 5 patients who met the study's inclusion criteria. Discrepancies were discussed to improve interpretation and application of the rubric, but no changes were made to the rubric itself. During the study period, every 20th chart evaluated for the study was audited by the same coinvestigator. Discrepancies were recorded to judge reliability of the rubric; in the event of a discrepancy, the chart was reviewed and the discrepancy resolved by consensus.

A score of $90 \%$ was established a priori as the threshold for competency in prescribing, based on the mark of $90 \%$ required to pass the RQHR certification exam for prescribing vancomycin. Each pharmacist received a score for his or her portion of the prescribing for each individual patient.

\section{Statistical Analysis}

An estimated competency rate of $85 \%$ was established based on the pass rate for the initial RQHR certification exam for vancomycin prescribing: a mark of $90 \%$ was required to pass the exam, and $85 \%$ of the pharmacists passed on their first attempt.
It was estimated that 200 patients would be required to detect this $85 \%$ competency rate. Given the estimated 100 patients per month for whom vancomycin was prescribed at the included facilities, the data collection period was set as November 1 to December 31, 2015, inclusive. Partway through the data collection period, it was determined that the required 200 patients would not be achieved in the predefined data collection period, and the period was therefore extended to January 31, 2016.

Data were assessed for normal distribution to determine whether parametric analysis could be performed. The data were not normally distributed, so medians and interquartile ranges (IQRs) were used to describe the data. Because of the non-normal distribution of the data, Mann-Whitney and Kruskal-Wallis tests were used to test for statistical significance in the secondary outcomes. All analyses were performed with IBM SPSS Statistics for Windows, version 22.0 (released 2013; IBM Corp, Armonk, New York). Statistical testing was not completed for competency score in relation to time of prescribing because of cross-over between the groups, representing non-independent observations.

\section{RESULTS}

In total, 326 patients received vancomycin in the study period; 200 of these, representing 511 discrete episodes of prescribing, were included in the study (Figure 1). Ten of the included patients had initial prescription of vancomycin by a

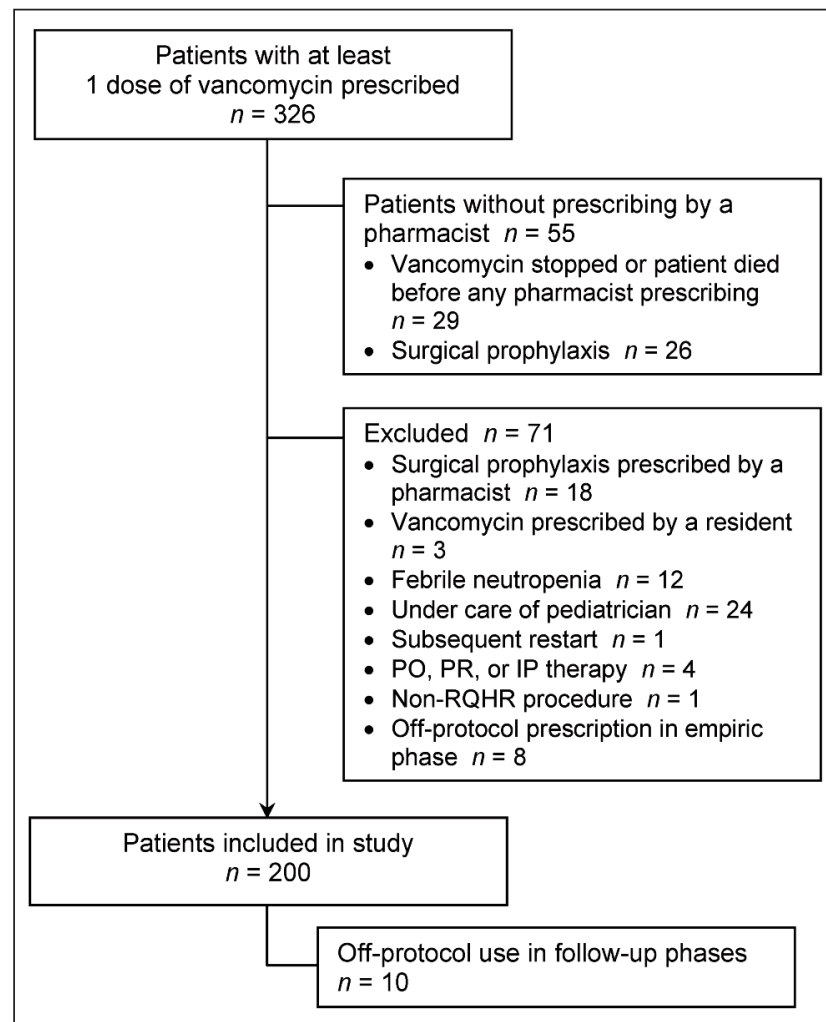

Figure 1. Identification of study sample. IP = intraperitoneal administration, $\mathrm{PO}=$ oral administration, $\mathrm{PR}=$ rectal administration, RQHR = Regina Qu'Appelle Health Region. 
pharmacist, but during the follow-up phase received "off-protocol" prescription of the drug. For those patients, only data before the "off-protocol" prescribing were included in the analysis.

Of the 63 pharmacists certified to prescribe vancomycin in the RQHR, 42 (67\%) had at least one phase of vancomycin prescribing included in the analysis (Table 1). Between 1 and 29 discrete episodes of prescribing were assessed for each individual pharmacist (median 12, IQR 5-18). Ten pharmacists (24\% of those included) had assessments for all phases of the vancomycin prescribing procedure. Pharmacist identity was unknown for 13 episodes of prescribing. These episodes were excluded from analyses of overall competency and competency in the monitoring and dose-adjustment phases, as well as the subgroup analyses based on pharmacist characteristics, but were included in the analyses based on patient characteristics (Table 2). Creatinine clearance as calculated during the assessment of empiric-phase prescribing was used for stratification on the basis of renal function. For 3 patients, vancomycin was discontinued by the pharmacist (because it was no longer indicated) before measurement of serum creatinine; these patients were therefore excluded from the analysis based on renal function. Four patients had crossover between locations (i.e., they appeared in both the critical care and non-critical care groups) and were therefore excluded from the secondary analysis based on patient location.

The median overall competency rate for all phases of vancomycin prescribing was $100 \%$ (IQR 90.1\%-100\%), with $32(76 \%)$ of the 42 pharmacists having overall competency scores of $90 \%$ or higher (Figure 2). The median levels of competency

Table 1. Characteristics of Pharmacists and Prescribing Episodes

\begin{tabular}{|c|c|c|}
\hline Characteristic & $\begin{array}{l}\text { No. (\%) of Pharmacists } \\
(n=42)\end{array}$ & $\begin{array}{l}\text { No. (\%) of Prescribing } \\
\text { Episodes }(n=498)^{*}\end{array}$ \\
\hline \multicolumn{3}{|l|}{ Phase of therapy } \\
\hline Empiric phase & 33 (78.6) & 185 (37.1) \\
\hline Monitoring or dose-adjustment phase & 39 (92.9) & $313(62.9)$ \\
\hline \multicolumn{3}{|l|}{ Pharmacist's experience at RQHR } \\
\hline$<2$ years & $13(31.0)$ & 195 (39.2) \\
\hline $2-5$ years & $10(23.8)$ & $128 \quad(25.7)$ \\
\hline$>5$ years & $19(45.2)$ & $175 \quad(35.1)$ \\
\hline \multicolumn{3}{|c|}{ Pharmacist's postbaccalaureate training } \\
\hline Yes & $19(45.2)$ & $221 \quad(44.4)$ \\
\hline No & $23(54.8)$ & $277 \quad(55.6)$ \\
\hline
\end{tabular}

* Of the 511 prescribing episodes included in the analysis, 13 were excluded from this table because the pharmacist's identity was unknown.

Table 2. Characteristics of Patients and Prescribing Episodes

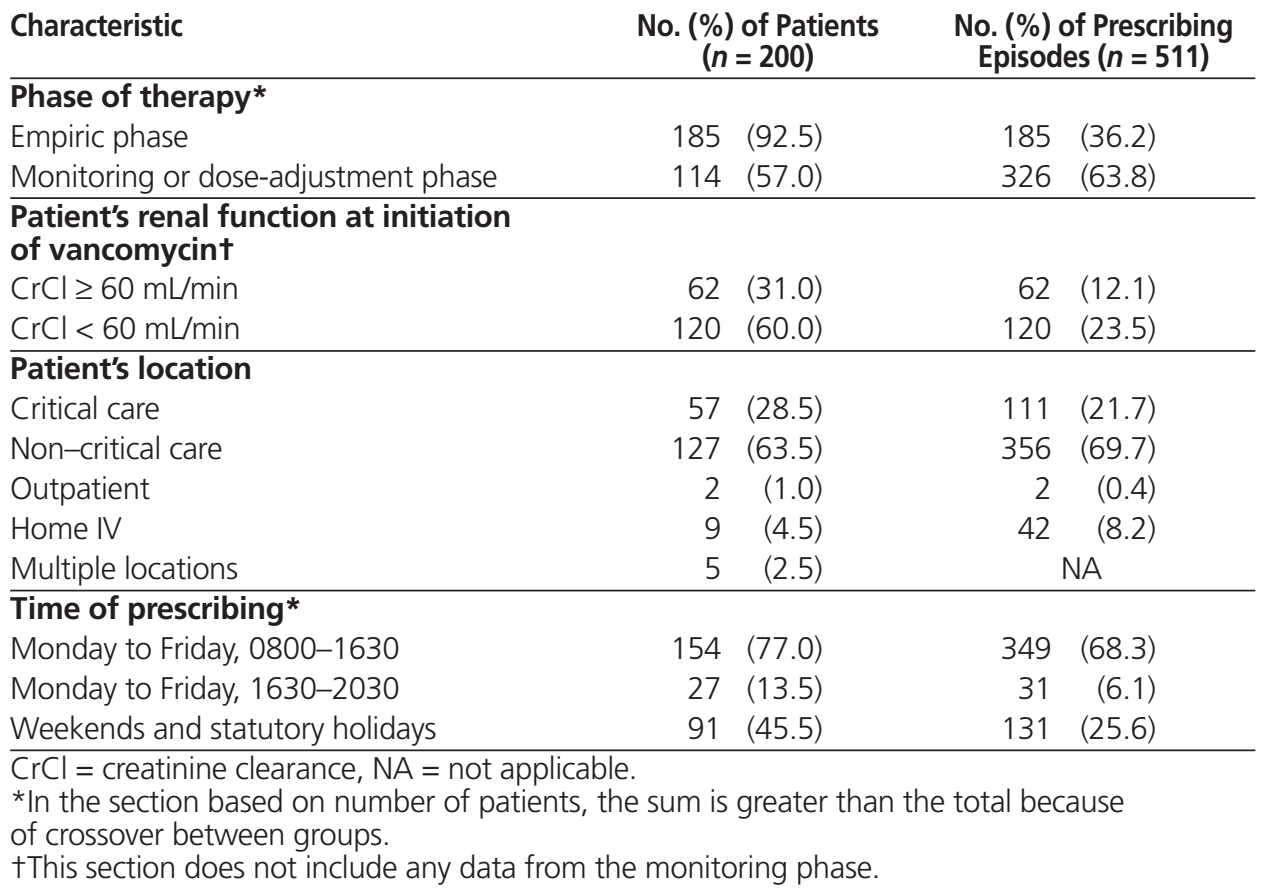




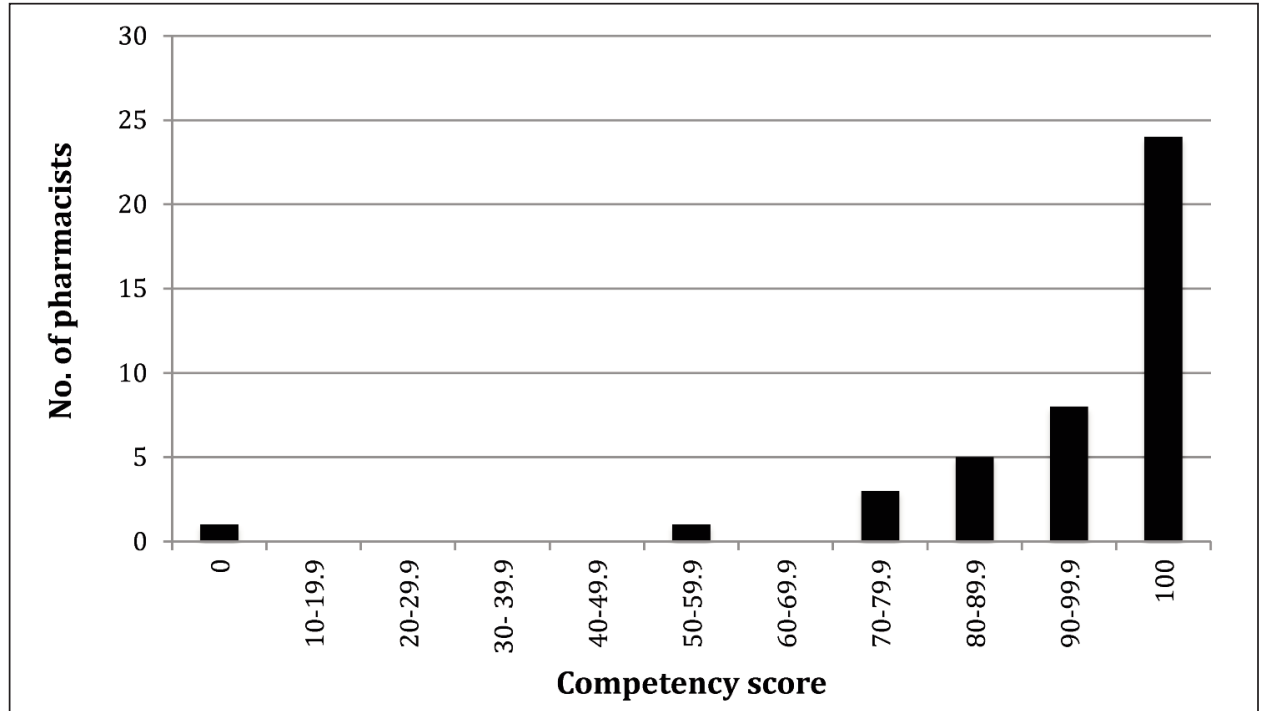

Figure 2. Pharmacists' overall competency scores.

for the empiric phase and monitoring phase were $94.4 \%$ (IQR $88.9 \%-100 \%$ ) and 100\% (IQR 87.5\%-100\%), respectively. Pharmacists' competency scores were higher when prescribing for patients with normal renal function than when prescribing for patients with reduced renal function (100\% [IQR 88.9\%-100\% versus $93.8 \%$ [IQR 76.1\%-100]; $p=0.008$ ). No statistically significant difference was found in relation to pharmacists' experience, pharmacists' postbaccalaureate education, or patients' level of acuity (as indicated by location of care) (Table 3).

Appendix 2 (available at www.cjhp-online.ca/index.php/ cjhp/issue/view/123/show Toc) details all of the 386 errors that were identified. The 3 most common prescribing errors were failure to document risk factors for nephrotoxicity (12\% of all errors [47/386]), which occurred in 47 (25\%) of the 185 empiric prescribing episodes; failure to document the requirement to obtain future trough levels (10\% of all errors [40/386]), which occurred in $40(26 \%)$ of the empiric prescribing episodes that applied to this area; and failure to document that samples for trough levels had been drawn correctly $(10 \%$ of all errors [37/386]). The critical errors of incorrect maintenance dose and incorrect dosing interval occurred in only 6 and 9 prescribing episodes, respectively.

For the 9 patients whose prescribing was assessed by both the primary investigator and the designated co-investigator, overall agreement was $86 \%$. This represented agreement on 31 of 36 discrete episodes of prescribing. Disagreements were related to timing of an ordered trough level, time at which prescribing occurred, and whether the pharmacist correctly documented when future levels would be required or if a trough level had been drawn correctly, among other differences. The chart for one additional patient was unavailable to the co-investigator, so some of this patient's episodes of prescribing were not compared.

\section{DISCUSSION}

The aim of this study was to assess the competency of pharmacists in their application of the current RQHR vancomycin protocol and procedure in a specified window of time. This assessment was achieved through development of a rubric and its application to records for patients with vancomycin prescribing by pharmacists. Although the rubric has not been internally validated, its application was audited throughout the study to increase the reliability of assessments. The agreement rate for selected records was $86 \%$, with only minor discrepancies noted, increasing our confidence in the data. External applicability is limited, because the rubric was created from a site-specific prescribing procedure.

Vancomycin prescribing was captured for two-thirds of eligible pharmacists, with an even distribution of experience and postbaccalaureate training. Although most prescribing episodes occurred during full staffing hours (0800-1630), prescribing during times of reduced staffing was also recorded. All practice areas and populations where the protocol was applicable were assessed. Unlike a previous study, ${ }^{9}$ which excluded patients with creatinine clearance less than $60 \mathrm{~mL} / \mathrm{min}$, this study included patients with normal and reduced renal function, which may increase its applicability across the populations for which pharmacists prescribe.

The median overall competency rate in this study showed that pharmacists in the RQHR are competent in empirically prescribing and monitoring vancomycin therapy, as well as adjusting vancomycin dosing, for patients with normal or impaired renal function in both critical care and non-critical care areas. The outpatient and home IV groups contained only small numbers of patients, which reduces our ability to draw conclusions about pharmacist competency for prescribing vancomycin for these subgroups. Patients who are critically ill have physiologic 
This single copy is for your personal, non-commercial use only.

For permission to reprint multiple copies or to order presentation-ready copies for distribution, contact CHHP at publications@cshp.ca

Table 3. Competency Rates in Relation to Pharmacist and Patient Characteristics

\begin{tabular}{|c|c|c|c|}
\hline Characteristic & $\begin{array}{r}\text { Med } \\
\mathbf{R}\end{array}$ & $\begin{array}{l}\text { an Competency } \\
\text { ate (\%) (IQR) }\end{array}$ & $p$ Value \\
\hline Phase of therapy & & & NA \\
\hline Overall & 100.0 & $(90.1-100.0)$ & \\
\hline Empiric phase & 94.4 & $(88.9-100.0)$ & \\
\hline Monitoring or dose-adjustment phase & 100.0 & $(87.5-100.0)$ & \\
\hline Pharmacist's experience at RQHR & & & 0.17 \\
\hline$<2$ years & 100.0 & $(95.7-100.0)$ & \\
\hline $2-5$ years & 100.0 & $(90.4-100.0)$ & \\
\hline$>5$ years & 92.9 & $(79.0-100.0)$ & \\
\hline Pharmacist's postbaccalaureate training & & & 0.17 \\
\hline No & 96.9 & $(88.2-100.0)$ & \\
\hline Yes & 100.0 & $(94.1-100.0)$ & \\
\hline Patient's renal function & & & 0.008 \\
\hline $\mathrm{CrCl} \geq 60 \mathrm{~mL} / \mathrm{min}$ & 100.0 & $(88.9-100.0)$ & \\
\hline $\mathrm{CrCl}<60 \mathrm{~mL} / \mathrm{min}$ & 93.8 & (76.1-100.0) & \\
\hline Patient's location & & & 0.10 \\
\hline Critical care & 100.0 & $(90.3-100.0)$ & \\
\hline Non-critical care & 94.4 & $(80.0-100.0)$ & \\
\hline Outpatient & 100.0 & $(100.0-100.0)$ & \\
\hline Home IV & 100.0 & $(90.9-100.0)$ & \\
\hline Time of prescribing & & & NA \\
\hline Monday to Friday, 0800-1630 & 94.4 & $(79.6-100.0)$ & \\
\hline Monday to Friday, 1630-2030 & 100.0 & $(88.9-100.0)$ & \\
\hline Weekends and statutory holidays & 93.9 & $(76.0-100.0)$ & \\
\hline
\end{tabular}

$\mathrm{CrCl}=$ creatinine clearance, $\mathrm{NA}=$ not applicable.

differences from the general patient population that could make vancomycin dosing and monitoring more challenging. These patients may have increased or decreased vancomycin clearance, often because of fluctuating renal function or changes in metabolic rates, making the correct maintenance dose unpredictable. ${ }^{3}$ Despite these complexities, the pharmacists in our study were competent in prescribing for this population. Patients with acute and chronic renal dysfunction have physiologic differences from those without renal dysfunction, including higher volume of distribution, decreased renal function (leading to an increase in vancomycin half-life), and requirement for dialysis, all of which can affect vancomycin management. ${ }^{3}$ Furthermore, the RQHR procedure contains a separate component for management of this population. Although a statistically significant difference in pharmacist competency was found in relation to renal function, the difference was not clinically significant, in that competency scores for prescribing for both subgroups were above the predefined acceptable level. There was no statistically significant difference based on years of experience or postbaccalaureate training, which suggests that these factors do not significantly affect the competency of a pharmacist in prescribing vancomycin.

Overall, $76 \%$ of the pharmacists received a competency score of $90 \%$ or greater and met the predetermined definition of competency. However, this proportion was lower than the hypothesized $85 \%$. The most common reasons for lost points were poor documentation or lack of documentation, rather than critical errors such as incorrect dose or dosing interval. The frequency and content of pharmacists documentation have previously been identified as areas for improvement. For example, Herritt and others ${ }^{13}$ found that pharmacists documented only $31 \%$ of their interventions.

Documentation is important for continuity of care within the health care team and should be reinforced. The most common errors in this study were failure to document risk factors for nephrotoxic risk factors, failure to document the requirement for future trough levels, and failure to document that samples for trough levels had been drawn correctly. Documenting risk factors for nephrotoxicity and the correct timing of a sample for determination of trough level are important to a pharmacist's clinical evaluation of a patient, and highlight important information for other members of the health care team. Documenting when future trough levels will be required helps to ensure that hand-off between pharmacists will not result in a delay in measuring levels. One possible explanation for the incomplete documentation in this study could be relative inexperience with the new protocol, as the frequently-made documentation errors related to new additions to the procedure. The new procedure, which replaced a procedure that had been in place for several decades, had been implemented for only 1.5 months before the start of the data collection period.

Several limitations may influence the interpretation of these results. The pharmacists were aware that the project was to take place, which may have affected their normal practices for prescribing and documenting. However, because of the retrospective 
design, they were not aware at the time of prescribing that their data might be included. With respect to documentation of appropriate timing of trough levels, if this was not done by the pharmacist, data collection was continued under the assumption that it had been done. This assumption was made because of an inability to retrospectively interpret the time at which samples were drawn in the absence of documentation. Because of the retrospective nature of this study, clinical interpretation of the available information could also have contributed to error (e.g., definition of deep-seated infection). All episodes of prescribing were treated equally, and there was no minimum number of episodes required for a pharmacist to be included in the study. For pharmacists with multiple episodes of prescribing, a mean score could be calculated for their prescribing, which was not the case for those with only one episode of prescribing; this difference may have affected overall competency scores. Lastly, this competency assessment represents a snapshot in time. Clinical practice is dynamic, and competency rates may change as pharmacists gain experience with the new procedure or because of new guideline recommendations.

This study has both internal and external implications. Externally, the rubric created for this study can be used as a template for assessment of pharmacist competency in other prescribing activities within RQHR and other health care delivery systems. Internally, the study data highlight a need to reinforce requirements for documentation and compliance with new procedures. Specifically, this project indicates the need to follow up with the $24 \%$ of pharmacists whose scores were below the predefined level of competency. The rates of competency in this study can serve as a benchmark for future competency assessments in the health region. As well, now that pharmacist competency with the vancomycin procedure has been confirmed, the RQHR can investigate the clinical outcomes of patients managed with this procedure.

\section{CONCLUSION}

RQHR pharmacists in various subgroups had an overall acceptable median level of competency in using the institution's vancomycin procedure for all phases of prescribing and across multiple pharmacist and patient groups during the study period. Documentation of clinical plans and assessments was identified as an area requiring further emphasis for the optimal management and collaborative care of this patient population.

\section{References}

1. Álvarez R, López Cortés LE, Molina J, Cisneros JM, Pachón J. Optimizing the clinical use of vancomycin. Antimicrob Agents Chemother. 2016;60(5): 2601-9.

2. Liu C, Bayer A, Cosgrove SE, Daum RS, Fridkin SK, Gorwitz RJ, et al. Clinical practice guidelines by the Infectious Diseases Society of America for the treatment of methicillin-resistant Staphylococcus aureus infections in adults and children. Clin Infect Dis. 2011;52(3):e18-55.
3. Burton ME, Shaw LM, Schentag JJ, Evans WE. Vancomycin. In: Applied pharmacokinetics and pharmacodynamics: principles of therapeutic drug monitoring. 4th ed. Baltimore (MD): Lippincott Williams \& Wilkins; 2006. p. 228-328.

4. Morrison AP, Melanson SEF, Carty MG, Bates DW, Szumita PM, Tanasijevic MJ. What proportion of vancomycin trough levels are drawn too early?: Frequency and impact on clinical actions. Am J Clin Pathol. 2012;137(3): 472-8.

5. Bassetti M, Righi E. Safety profiles of old and new antimicrobials for the treatment of MRSA infections. Expert Opin Drug Saf. 2016;15(4):467-81.

6. van Hal SJ, Paterson DL, Lodise TP. Systematic review and meta-analysis of vancomycin-induced nephrotoxicity associated with dosing schedules that maintain troughs between 15 and 20 milligrams per liter. Antimicrob Agents Chemother. 2013;57(2):734-44.

7. Rybak MJ, Lomaestro BM, Rotschafer JC, Moellering RC Jr, Craig WA, Billeter M, et al. Vancomycin therapeutic guidelines: a summary of consensus recommendations from the Infectious Diseases Society of America, the American Society of Health-System Pharmacists, and the Society of Infectious Diseases Pharmacists. Clin Infect Dis. 2009;49(3):325-7.

8. Szeto J, Brown G. Impact of collaborative drug therapy management by pharmacists [letter]. Can J Hosp Pharm. 2002;55(5):333-6.

9. Marquis KA, DeGrado JR, Labonville S, Kubiak DW, Szumita PM. Evaluation of a pharmacist-directed vancomycin dosing and monitoring pilot program at a tertiary academic medical center. Ann Pharmacother. 2015; 49(9):1009-14.

10. Masuda N, Maiguma T, Komoto A, Haruki Y, Sugiyama T, Kondo S, et al. Impact of pharmacist intervention on preventing nephrotoxicity from vancomycin. Int J Clin Pharmacol Ther. 2015;53(4):284-91.

11. McCormack J, Cooper J, Carleton B. Simple approach to dosage adjustments in patients with renal impairment. Am J Health Syst Pharm. 1997;54(21): 2505-9.

12. Wilhelm SM, Kale-Pradhan PB. Estimating creatinine clearance: a metaanalysis. Pharmacotherapy. 2011;31(7):658-64.

13. Herritt L, MacInnis M, Grimm N, Lee R, MacLean D, MacNeil T, et al. Analysis of current pharmacist documentation practices in a tertiary care hospital [abstract]. Can J Hosp Pharm. 2014;67(4):318.
Kirsten Tangedal, BSP, ACPR, is with the Pharmacy Department, Regina Qu'Appelle Health Region, Regina, Saskatchewan.

Jennifer Bolt, BSCPharm, ACPR, PharmD, is with the Pharmacy Department, Regina Qu'Appelle Health Region, Regina, Saskatchewan.

Suzanne Len, BSP, ACPR, with the Pharmacy Department, Regina Qu'Appelle Health Region, Regina, Saskatchewan.

Ali Bell, MA, MSc, is with Research \& Performance Support, Regina Qu'Appelle Health Region, Regina, Saskatchewan.

Competing interests: None declared.

\section{Address correspondence to:}

Kirsten Tangedal

Pharmacy Department

Regina Qu'Appelle Health Region

1440 14th Avenue

Regina SK S4P OW5

e-mail: kirsten.tangedal@rqhealth.ca

Funding: None received. 